\title{
Kaposi's Sarcoma-Associated Herpesvirus Infection in the Lung in Multicentric Castleman's Disease
}

\author{
Mitsutoshi Hayashi, Kazutetsu Aoshiba, Masahiko Shimada, Yutaka Izawa, \\ Shuji Yasui and Atsushi NaGaI
}

\begin{abstract}
A 32-year-old female was admitted for evaluation of multiple infiltrates on a chest radiograph. A diagnosis of multicentric Castleman's disease was made on the basis of typical clinical manifestations. Transbronchial lung biopsy (TBLB) revealed histological findings reported in lymphocytic interstitial pneumonia. Both the polymerase chain reaction and in situ hybridization with a probe specific for Kaposi's sarcoma-associated herpesvirus (KSHV) sequences demonstrated the presence of KSHV in the TBLB sample.
\end{abstract}

(Internal Medicine 38: 279-282, 1999)

Key words: lymphocytic interstitial pneumonia, interleukin-6, transbronchial lung biopsy

\section{Introduction}

Multicentric Castleman's disease (MCD) is a rare lymphoproliferative disorder characterized by systemic lymphadenopathy, multiple organ involvement, polyclonal hypergammaglobulinemia, and other nonspecific clinical manifestations such as fever, anemia, an elevated erythrocyte sedimentation rate, and an increased serum interleukin-6 (IL-6) level (1). Epidemiologic studies suggest a close association between MCD and Kaposi's sarcoma, which occurs during the clinical course of MCD in $13 \%-75 \%$ of cases $(1,2)$. This epidemiologic finding is further supported by the recent identification of a new herpesvirus, Kaposi's sarcoma-associated herpesvirus (KSHV), also known as human herpesvirus-8 (HHV-8), in samples obtained from both Kaposi's sarcoma and from MCD $(2-4)$.

For editorial comment, see also p 221.

Although little is known with regard to the pulmonary lesions of MCD, a case of MCD with lymphocytic interstitial pneumonia (LIP) has been documented (5). Here, we report a case of MCD with lymphoproliferative lesions in the lung in which KSHV infection was demonstrated.

\section{Case Report}

A 32-year-old HIV-negative Japanese female was admitted to our hospital for evaluation of abnormal shadows on a routine chest radiograph. She was asymptomatic, and physical examination showed no abnormalities except for left supraclavicular and bilateral axillar lymphadenopathy. Laboratory data disclosed normocytic anemia of $9.3 \mathrm{~g} / \mathrm{dl}$ hemoglobin, an elevated erythrocyte sedimentation rate of $128 \mathrm{~mm} / \mathrm{h}$, hypoalbuminemia of $2.7 \mathrm{~g} / \mathrm{dl}$, and a polyclonal hypergammaglobulinemia of $5.9 \mathrm{~g} / \mathrm{dl}$, mostly composed of increases in immunoglobulin G (IgG). Serum IL-6 was increased to 13.1 $\mathrm{pg} / \mathrm{dl}$ (normal range: $<4 \mathrm{pg} / \mathrm{dl}$ ). Liver and renal function tests were normal. A plain film and computed tomography of the chest (Fig. 1) showed multiple hazy infiltrates in both lung fields, with interseptal thickening and mediastinal lymphadenopathy. Pulmonary function studies were normal, and bacterial cultures of sputum and sputum cytology were negative. Broncho-fiberscopy with bronchoalveolar lavage demonstrated a mild lymphocytosis of $9.1 \%$, with the B-cell (CD20+) fraction increased to $18.2 \%$. Transbronchial lung biopsy (TBLB) (Fig. 2) revealed infiltration of lymphocytes, macrophages, and fewer plasma cells, with formation of lymphoid follicles in the alveolar and peribronchiolar interstitium, which is similar to the histological findings reported in LIP (6). Immunohistochemical staining of the TBLB specimen was positive for both $\kappa$ and $\lambda$ immunoglobulin, indicating polyclonal proliferation of lymphocytes and plasma cells in the lung (Fig. 2B, C). These histologic findings together with the presence of clinical features such as multicentric lymphadenopathy, polyclonal hypergammaglobulinemia, an elevated erythrocyte sedimen-

From the Department of Medicine, Chest Institute Tokyo Women's Medical University, Tokyo

Received for publication June 9, 1998; Accepted for publication December 12, 1998.

Reprint requests should be addressed to Dr. Atsushi Nagai, the Department of Medicine, Tokyo Women's Medical University, 8-1 Kawada-cho, Shinjuku-ku, Tokyo 162-8666 


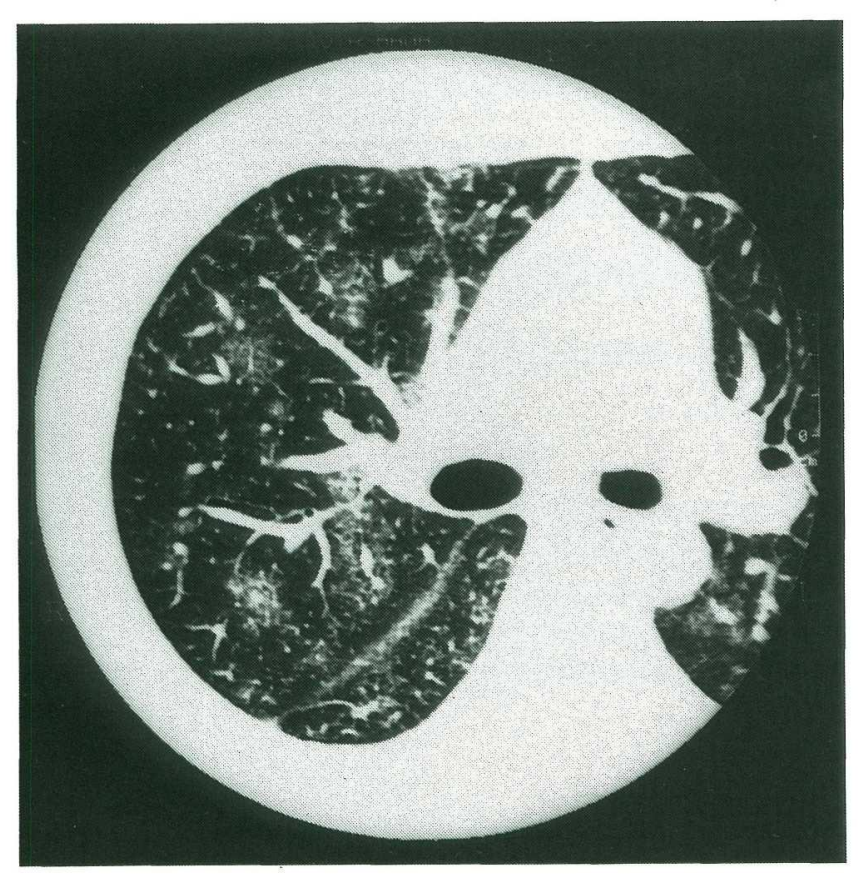

Figure 1. Computed tomogram of the chest showing multiple alveolar infiltrates with interseptal thickening.

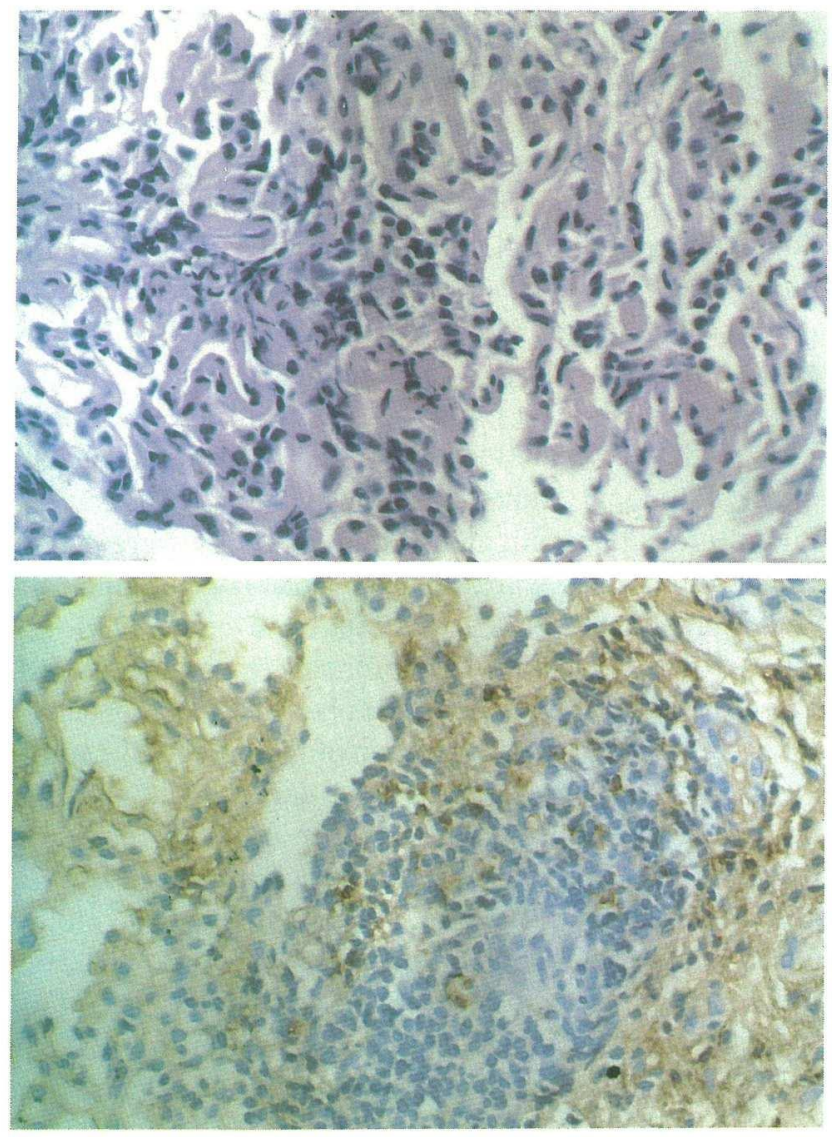

tation rate, and an increased serum IL-6 level were the basis for making the diagnosis of MCD.

To investigate the etiology, the TBLB specimen was analyzed for KSHV. Polymerase chain reaction (PCR) was performed as described previously (7) to detect the $\mathrm{KS} 330_{233}$ sequence, which represents a 233-bp fragment of the KS330 Bam region of KSHV (3). Briefly, $100 \mathrm{ng}$ of genomic DNA was extracted from the sample and a total of 35 cycles of PCR amplification was performed using the following primers: forward primer, 5'-AGCCGAAAGGATTCCACCAT-3'; reverse primer, $5^{\prime}$-TCCGTGTTGTCTACGTCCAG-3' $(3,7)$. The PCR product was electrophoresed on a $1 \%$ agarose gel containing ethidium bromide and photographed. As shown in Fig. 3, the $\mathrm{KS} 330_{233}$ sequence was detected in the lung specimen obtained from this patient, but not in the lung specimen obtained from a control subject having an irrelevant lung disease. We also performed dot blotting of the PCR amplification product with a ${ }^{32} \mathrm{p}$ end-labeled probe internal to the $\mathrm{KS} 330_{233}$ sequence $5^{\prime}$ TGCAGCAGCTGTTGGTGTACCACA-3' as described (8) and found that the PCR product specifically hybridized with the internal probe (data not shown).

In situ hybridization was performed as another confirmatory technique to document the specificity of the KSHV infection of
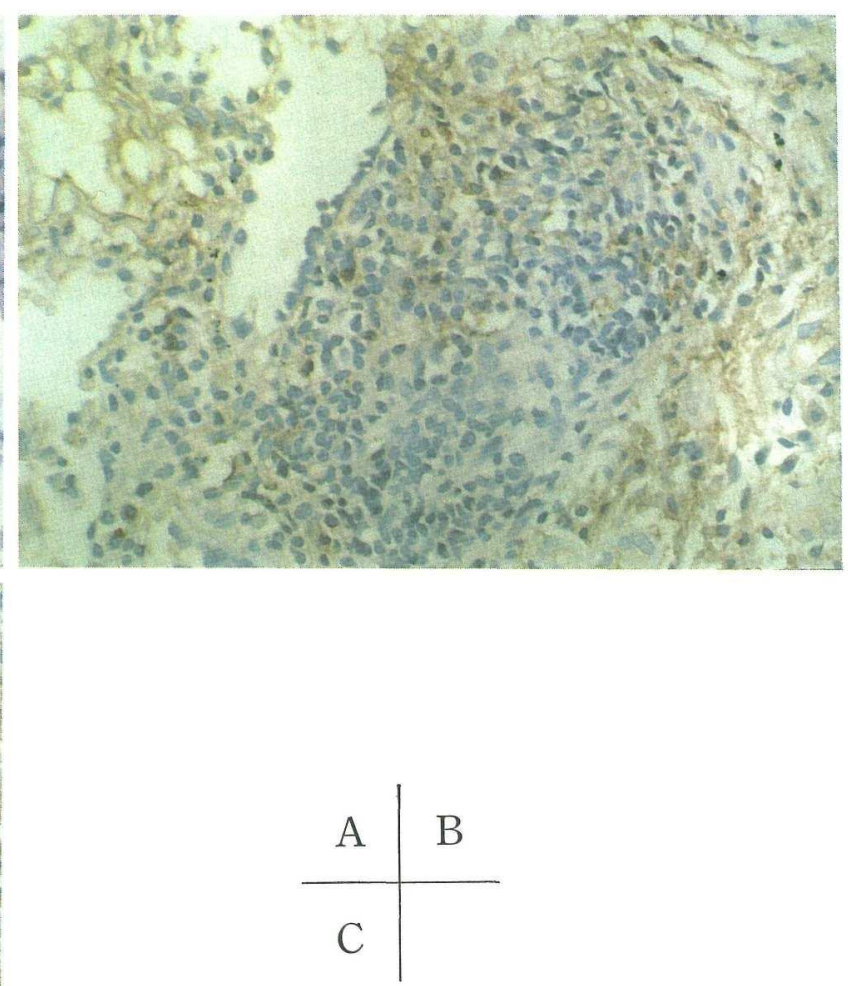

Figure 2. Light micrograph of TBLB $(\times 100)$. A. HE staining showing infiltration by lymphocytes, macrophages, and fewer plasma cells in the alveolar interstitium. B and C. Hyperplasia of a lymphoid follicle that has immunostained positive for both $\kappa(B)$ and $\lambda(\mathbf{C})$ gammaglobulins. 
1
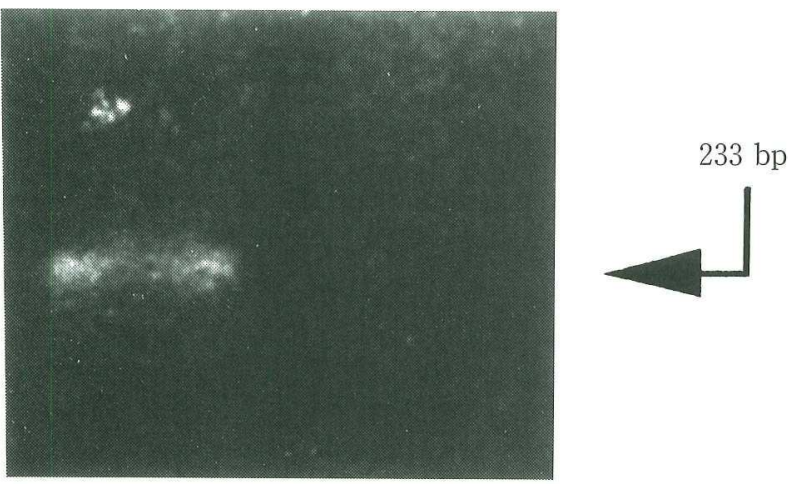

Figure 3. Agarose gel electrophoregram of the PCR amplification product. The $\mathrm{KS}_{330}{ }_{233} \mathrm{PCR}$ product is detected in the TBLB from the patient (lane 1 ) but not in the TBLB from a control subject (lane 2).

the lung. Paraffin-embedded TBLB sections were mounted on ProbeOn glass slides ${ }^{\mathrm{TM}}$ (Fisher Scientific, Pittsburgh, PA), and in situ hybridization was performed using the MicroProbe ${ }^{\circledR}$ system (Fisher) as described preiviously (9). The oligonucleotide probe was in sense orientation with the following sequence: $5^{\prime}$ TGCAGCAGCTGTTGGTGTACCACATCTACT-3' linked to a $3^{\prime}$-biotinylated tail [3'-(TAG) $)_{5}$-BBB-(TAG) $\left.)_{2}-\mathrm{BBB}-5^{\prime}\right]$ (7). In situ hybridization with the KSHV probe demonstrated nuclear and cytoplasmic staining (7) of some cells infiltrating the pulmonary interstitium (Fig. 4). Either omission of the KSHV probe or replacement of the probe with an irrelevant oligonucleotide probe (rat IL-6 probe) resulted in negative staining. These KSHV-positive cells, although not identified, appear to represent macrophages due to their relatively large size. Immunohistochemical and ultrastructural studies to identify these cells could not be performed.

\section{Discussion}

In the present case, a diagnosis of $\mathrm{MCD}$ was made primarily on the basis of typical clinical manifestations including multifocal lymphadenopathy, polyclonal hypergammaglobulinemia with hypoalbuminemia, an elevated erythrocyte sedimentation rate, and an increased IL-6 level. Thus, it should be noted that the diagnosis of MCD may not be definite because lymph node biopsy, which is essential for histological diagnosis of MCD, could not be performed.

$\mathrm{MCD}$ is a rare lymphoproliferative disorder that involves multiple organs, in addition to lymph nodes (1), but little attention has been paid to involvement of the lung. We have described a case having typical clinical manifestations of MCD and involving the lung which showed histological pictures similar to that of LIP. LIP can occur idiopathically, in association with Sjören's syndrome and other autoimmune diseases, and in people infected with human immunodeficiency virus (HIV) (6). The present case suggests that pulmonary lesions resem-

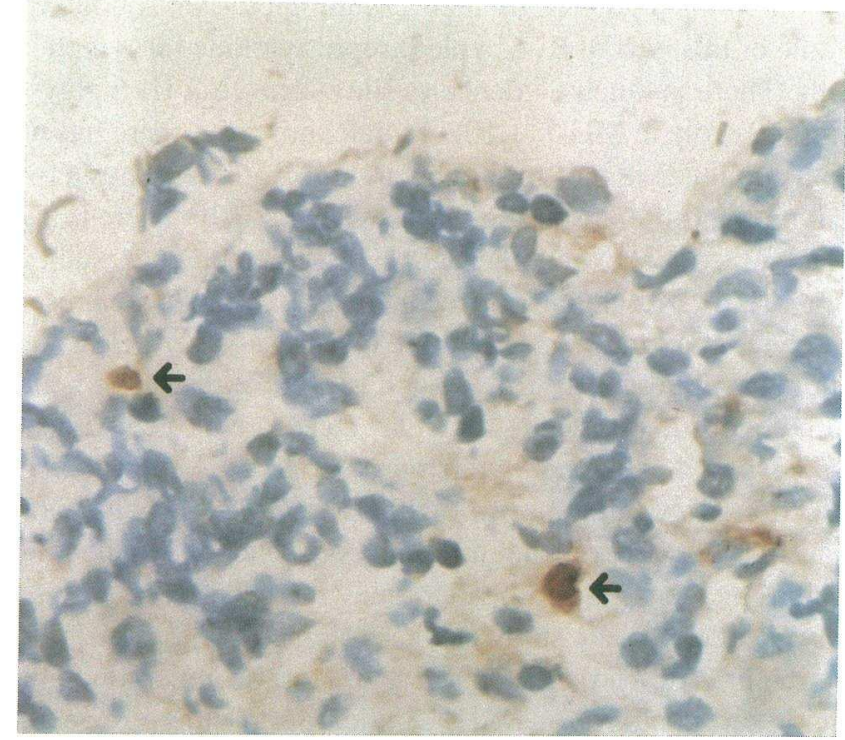

Figure 4. In situ hybridization of a biotinylated KSHV probe, showing nuclear and cytoplasmic staining of some cells infiltrating cells (arrows) in TBLB (original magnification $\times 200$ )

bling LIP may also occur as a manifestation of MCD. Although little is known with regard to pulmonary lesions of $\mathrm{MCD}$, a recent report has also described a case of MCD with pulmonary lesions resembling LIP(5).

We also detected evidence of KSHV infection in the lung lesion. KSHV, a gamma herpes virus related to Epstein-Barr virus, was first identified in acquired immunodeficiency syndrome (AIDS)-associated Kaposi's sarcoma (3), but was later detected in lymph nodes of MCD as well (2). To our knowledge, our report is the first to document detection of KSHV in an extranodal organ in MCD. It might be suggested that leukocytes circulating in the pulmonary vasculature were the source of the PCR product we detected. However, in situ hybridization demonstrated that KSHV-positive cells were located in the extravascular space of the pulmonary interstitium.

Although we detected evidence of KSHV infection in the lung, it remains unclear whether KSHV is responsible for the pulmonary lesion found in this case. We cannot exclude the possibility that KSHV infection is incidental and irrelevant to the pathogenesis of the pulmonary lesion. Nevertheless, recent evidence suggests that KSHV may play a role in the pathogenesis of lymphoproliferative disorders. KSHV sequences share homologies with many cellular genes, including host genes known to function in cell growth (cyclin D), apoptosis (bcl-2), cell-cell interaction (OX-2), immunoregulation, and cytokine signaling (IL-6, CC chemokines, CXC chemokine receptors) (10). It therefore seems reasonable to suppose that cytokines, cytokine receptors, and growth regulatory proteins encoded by these viral genes support the proliferation and activation of lymphoid cells in KSHV-infected tissues. In fact, many of these viral genes have been shown to encode proteins that preserve 


\section{HAYASH et al}

the functions predicted by their homologies (10). A good example of this is viral IL-6, a pleiotropic cytokine for lymphoid cells. There is much evidence to show that viral IL-6 plays a causative role in $\operatorname{MCD}(1,10)$. To date, however, there has not been enough evidence to show that viral IL-6 also plays a role in lymphoproliferative lung diseases such as LIP. There is also a lack of evidence that other KSHV-associated genes, such as viral bcl-2, CXC chemokine receptor, and cyclin $\mathrm{D}$, are present in the lung in lymphoproliferative diseases. Nevertheless, our findings suggest that viruses such as KSHV that have genes homologous to host genes may play a causative role in at least some types of pulmonary lymphoproliferative disorders. KSHV infection of the lung may allow the virus to express its genes that support lymphoid and inflammatory reactions in the lung, such as the genes encoding cytokines and cell growth.

An epidemiologic study using serologic tests for KSHV infection has shown that at least $1 \%-2 \%$ of the general population is seropositive for KSHV, indicating past exposure and ongoing latency in at least some host cells (10). Our findings may provide a clue to the pathogenesis of lymphoproliferative lung diseases, whose cause needs to be clarified. Given the highly unusual biology of KSHV, it is important to search for further evidence of pulmonary infection with KSHV.

\section{References}

1) Peterson BA, Frizzera G. Multicentric Castleman's disease. Semin Oncol
20: 636-647, 1993.

2) Soulier J, Grollet L, Oksenhendler E, et al. Kaposi's sarcoma-associated herpesvirus-like DNA sequences in multicentric Castleman's disease. Blood 86: 1276-1280, 1995.

3) Chang Y, Cesarman E, Pessin MS, et al. Identification of herpesvirus-like DNA sequences in AIDS-associated Kaposi's sarcoma. Science 266: 18651869, 1994.

4) Moore PS, Chang Y. Detection of herpesvirus-like DNA sequences in Kaposi's sarcoma in patients with and without HIV infection. N Engl J Med 332: 1181-1185, 1995.

5) Sato T, Wakabayashi Y, Hirasawa A, Tashiro M, Nishikawa T, Chiba S. A case of multicentric Castleman's disease accompanied with both lymphoid interstitial pneumonia and interstitial nephritis. Rinsho Ketsueki (Jpn J Clin Hematol) 35: 1322-1328, 1994.

6) King Jr TE, Cherniack RM, Schwartz MI. Idiopathic pulmonary fibrosis and other interstitial lung diseases of unknown etiology. in: Textbook of Respiratory Medicine, 2nd ed. Murray JF, Nadel JA, Eds. W. B. Saunders Company, Philadelphia, 1994: 1827-1849.

7) Rettig MB, Ma HJ, Vescio RA, et al. Kaposi's sarcoma-associated herpes virus infection of bone marrow dendritic cells from multiple myeloma patients. Science 276: 1851-1854, 1997.

8) Brown T. Dot and slot blotting of DNA. in: Short Protocols in Molecular Biology, Ausubel FM, Brent R, Kingston RE, Moore DD, Seidman JG, Smith JA, Struhl K, Eds. 3rd ed, John Wiley \& Sons, Inc., 1995: p2-33p2-35.

9) Wang JY, Montone KT. A rapid simple in situ hybridization method for herpes simplex virus employing a synthetic biotin-labeled oligonucleotide probe: a comparison with immunohistochemical methods for HSV detection. J Clin Lab Anal 8: 105-115, 1994.

10) Ganem D. KSHV and Kaposi's sarcoma: The end of the biginning? Cell 91: 157-160, 1997. 